\title{
High-speed, energy-resolved STJ observations of the AM Her system V2301 Oph
}

\author{
A. P. Reynolds ${ }^{1}$, G. Ramsay ${ }^{2}$, J. H. J. de Bruijne ${ }^{1}$, M. A. C. Perryman ${ }^{1}$, M. Cropper ${ }^{2}$, \\ C. M. Bridge ${ }^{2}$, and A. Peacock ${ }^{1}$ \\ 1 Research and Scientific Support Department of ESA, ESTEC, Postbus 299, 2200 AG Noordwijk, The Netherlands \\ ${ }^{2}$ Mullard Space Science Laboratory, University College London, Holmbury St Mary, Dorking, Surrey RH5 6NT, UK
} e-mail: mperryma@rssd.esa.int

Received 11 December 2003 / Accepted 23 January 2005

\begin{abstract}
We present high time-resolution optical energy-resolved photometry of the eclipsing cataclysmic variable V2301 Oph made using the ESA S-Cam detector, an array of photon counting super-conducting tunnel junction (STJ) devices with intrinsic energy resolution. Three eclipses were observed, revealing considerable variation in the eclipse shape, particularly during ingress. The eclipse shape is shown to be understood in terms of AM Her accretion via a bright stream, with very little contribution from the white dwarf photosphere and/or hotspot. About two thirds of the eclipsed light arises in the threading region. Variation in the extent of the threading region can account for most of the variations observed between cycles. Spectral fits to the data reveal a $10000 \mathrm{~K}$ blackbody continuum with strong, time-varying emission lines of hydrogen and helium. This is the first time that stellar emission lines have been detected in the optical band using a non-dispersive photon-counting system.
\end{abstract}

Key words. binaries: eclipsing - instrumentation: detectors - stars: individual: V2301 Oph - white dwarfs

\section{Introduction}

V2301 Oph is an interacting binary system with an orbital period of 113 min (Silber et al. 1994). The spectropolarimetric study of Ferrario et al. (1995), made during a low accretion state, established that its accreting white dwarf has a magnetic field strength of $B \sim 7$ MG. On this basis, V2301 Oph is a polar (a magnetic cataclysmic variable, $\mathrm{CV}$ ), with the weakest magnetic field of any member of this class. In these systems, matter flows from the donor star along a ballistic stream, until it reaches a "threading region" where it becomes attached to the magnetic field of the white dwarf. The material then travels along the field lines, until it forms a column of material settling onto the surface of the white dwarf via a shock (e.g. Ferrario $\&$ Wehrse 1999). In some systems (e.g. HU Aqr, Bridge et al. 2002) the details of the accretion geometry can be reliably related to the shape of the eclipse lightcurve. The highly variable nature of the eclipse profiles in V2301 Oph, however, make the interpretation of the lightcurve more challenging.

With its unique combination of high time-resolution and energy discrimination, S-Cam2 - ESA's energy-sensitive photon-counting camera - has proven particularly useful as a tool for studying the rapidly varying optical emission of CVs. Technical descriptions of the instrument may be found in Rando et al. $(1998,2000)$, while the data acquisition and reduction methods are outlined in Perryman et al. $(1999,2001)$. In our previous studies of CVs (Perryman et al. 2001; Bridge et al. 2002, 2003; Steeghs et al. 2003) our main tool for
Table 1. Observations of V2301 Oph obtained using S-Cam2. The ephemeris of Barwig et al. (1994) has been used to determine the binary orbital phases.

\begin{tabular}{ccccc}
\hline \hline No. & $\begin{array}{c}\text { Date } \\
(2000)\end{array}$ & Cycle & $\begin{array}{c}\text { Obs. } \\
\text { (UTC) }\end{array}$ & Phase range \\
\hline 1 & April 27 & $45769-45770$ & $03: 52-04: 28$ & $0.802-1.115$ \\
2 & April 28 & $45781-45782$ & $02: 17-03: 03$ & $0.706-1.106$ \\
3 & April 29 & $45793-45794$ & $01: 03-01: 43$ & $0.800-1.154$ \\
\hline
\end{tabular}

understanding the spectral changes has been energy-selected lightcurves and their associated colour ratios. Here, we present S-Cam2 data of V2301 Oph and relate the light curves to previous observations and demonstrate that S-Cam2 can detect and quantify contributions from individual emission lines of hydrogen and helium.

\section{Observations}

The S-Cam2 campaigns were performed during April 2000 at the 4.2-m William Herschel Telescope on La Palma, with the instrument mounted at the Nasmyth focus: the seeing was variable, ranging from good to poor. The observations were planned so as to include three complete eclipses, with representative data either side. Due to constraints from other program targets, it was not possible to observe an entire cycle. The observations are logged in Table 1. We estimate that V2301 Oph was at $V \sim 15.7$, indicating it was in a high accretion state. 

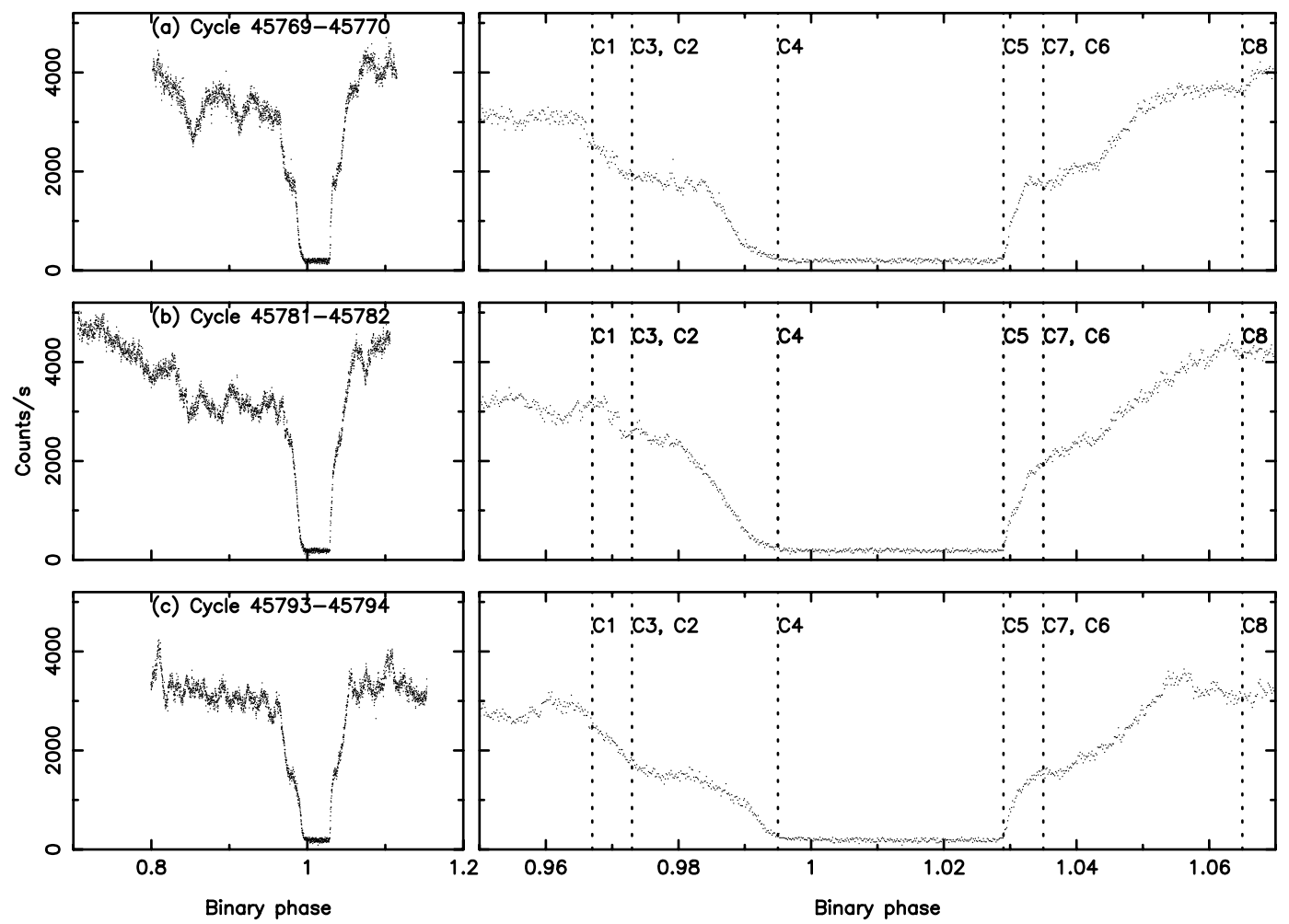

Fig. 1. Phase-folded light curves of the three V2301 Oph observations. The right-hand plots are enlargements of the eclipse intervals in the left-hand plots. These white-light $(\lambda \sim 3200-7200 \AA)$, background-subtracted light curves have been corrected for variations in pixel-to-pixel gain and responsivity. A time (i.e., zenith angle) dependent correction for atmospheric extinction has also been applied. The data have been binned into $1 \mathrm{~s}$ intervals. The phases and cycle numbers are with respect to the ephemeris of Barwig et al. (1994). The vertical dashed lines indicate the phases of the contact points identified by Steiman-Cameron \& Imamura (1999); $c_{1}$ and $c_{5}$ are the contact points of photospheric ingress and egress respectively.

In Fig. 1 we show "white-light" lightcurves for the three observations, after barycentric correction, background subtraction, phase-folding and correction for atmospheric extinction; see Perryman et al. (2001) for a description of these data reduction steps. The data have also been corrected for variations in pixel-to-pixel detection efficiency and energy response. The light curves show that the accretion stream in V2301 Oph is particularly prominent in the optical band, compared with many other polars.

\section{Light curves}

Our data were phased with respect to the orbital ephemeris of Barwig et al. (1994), which was also the ephemeris used by Steiman-Cameron \& Imamura (1999). There are significant variations between the 3 eclipse profiles in each of the eclipses. They do, however, exhibit a similar shape to those seen in other studies. The first is similar to the 1997 June 2 eclipse seen by Steiman-Cameron \& Imamura (1999), with a pronounced two-stepped descent into the eclipse. This two-stepped profile is also seen in the UV data of Schmidt \& Stockman (2001).

Steiman-Cameron \& Imamura (1999) identify eight contact points in the lightcurve, associated with the ingress/egress of the white dwarf photosphere and the ingress/egress of the accretion stream. By reference to Fig. 6 in Steiman-Cameron \& Imamura, we determined the phases of their contact points and then plotted these phase points as vertical dashed lines in our Fig. 1. They associate $c_{1}, c_{2}, c_{5}$ and $c_{6}$ with the eclipse of the photosphere, while they associate the four other contact points with the eclipse of the accretion stream. We show the observed times of these contact points in Table 2 .

The contact points as defined by Steiman-Cameron \& Imamura (1999) generally match up with the same feature in our light curves. For contact points $\mathrm{c}_{4,5}$ the features line up very well. There is some discrepancy in the eclipse ingress because the accretion stream in V2301 Oph is relatively bright compared to many other polars. In the second eclipse, the stream accounts for roughly $3 / 4$ of the optical emission. We believe that discrepancies between the other contact points can be explained by a variation in the amount of material located in different regions of the accretion stream trajectory. We discuss this in greater depth in Sect. 5.

\section{Spectral properties}

The intrinsic energy sensitivity of the S-Cam2 detector permits high time-resolution spectrophotometry of astronomical sources. The resulting data can be analysed in terms of energy (colour) bands and their associated ratios (Perryman et al. 2001) or even direct spectral fitting (de Bruijne et al. 2002; Reynolds et al. 2003). In this paper we extend this spectralfitting to accommodate the presence of emission lines, a 
Table 2. The times for the contact points for the three eclipses. The times are in MJD. The error on each time estimate is $\sim 3 \mathrm{~s}$.

\begin{tabular}{lcccc}
\hline \hline $\begin{array}{l}\text { Contact } \\
\text { points }\end{array}$ & Phase & Eclipse 1 & Eclipse 2 & Eclipse 3 \\
\hline C1 & $(0.967)$ & 51661.17442 & 51662.11582 & 51663.05722 \\
C2, C3 & $(0.973)$ & 51661.17489 & 51662.11629 & 51663.05769 \\
C4 & $(0.995)$ & 51661.17661 & 51662.11802 & 51663.05942 \\
C5 & $(1.029)$ & 51661.17929 & 51662.12069 & 51663.06209 \\
C6, C7 & $(1.035)$ & 51661.17975 & 51662.12116 & 51663.06255 \\
C8 & $(1.065)$ & 51661.18211 & 51662.12350 & 51663.06491 \\
\hline
\end{tabular}

particularly useful technique for cataclysmic variables due to the swiftness of the eclipse transitions. First, however, we examine the data in terms of energy bands, since this is useful for characterising broad changes in emission properties.

\subsection{Colour bands}

The data were segregated into three bands corresponding to blue $(3200-4700 \AA)$, visual $(4700-5500 \AA)$ and red (5500-7200 $\AA$ colours). We label these bands $B^{\prime}, V^{\prime}$ and $R^{\prime}$, emphasising that they are not on any formal photometric system. The boundaries between the bands are selected to ensure that roughly equal numbers of events are assigned to each band. In Fig. 2, we again show the three eclipses, but this time split into these three energy-selected lightcurves. The amplitudes of the $V^{\prime}$ and $B^{\prime}$ band curves are adjusted slightly to correct for small differences in total counts (the initial segregation only giving approximate equipartition among events) and the $V^{\prime}$ and $B^{\prime}$ bands are then offset vertically on the same plots. Differences in slope between the bands are therefore meaningful. Although there are no dramatic differences between the bands, in all three eclipses the $R^{\prime}$ band data exhibits noticeably more structure, with the steps and the ledges between them more sharply defined than in the other bands. In particular, the first and third eclipses both contain dips in the $R^{\prime}$ band at phase $\sim 0.0375$, which are much less apparent in the $B^{\prime}$ and $V^{\prime}$ data. In the first eclipse, as well, the ledge between the two ingress steps is more or less horizontal, whereas it shows a pronounced slope in the $B^{\prime}$ and $V^{\prime}$ data. This suggests that if the material responsible for the steps is concentrated in two distinct regions, then the region between them is emitting mainly at shorter wavelengths than that seen in the $R^{\prime}$ band data, and/or is preferentially absorbed in the red.

The relatively small numbers of events per time bin in each colour band make the study of colour ratios difficult. In order to remove short-term variations due to seeing, guiding errors and intrinsic flickering, we first smoothed the individual $R^{\prime}$ and $B^{\prime}$ band lightcurves using a sliding cell with a width of $15 \mathrm{~s}$. The ratio $R^{\prime} / B^{\prime}$ against binary phase, for all three eclipses, is shown in the narrower panels in Fig. 2. It is apparent that there are significant differences in the variation of $R^{\prime} / B^{\prime}$ between the three observations, particularly in the late stages of the ingress, just before the onset of deep eclipse.
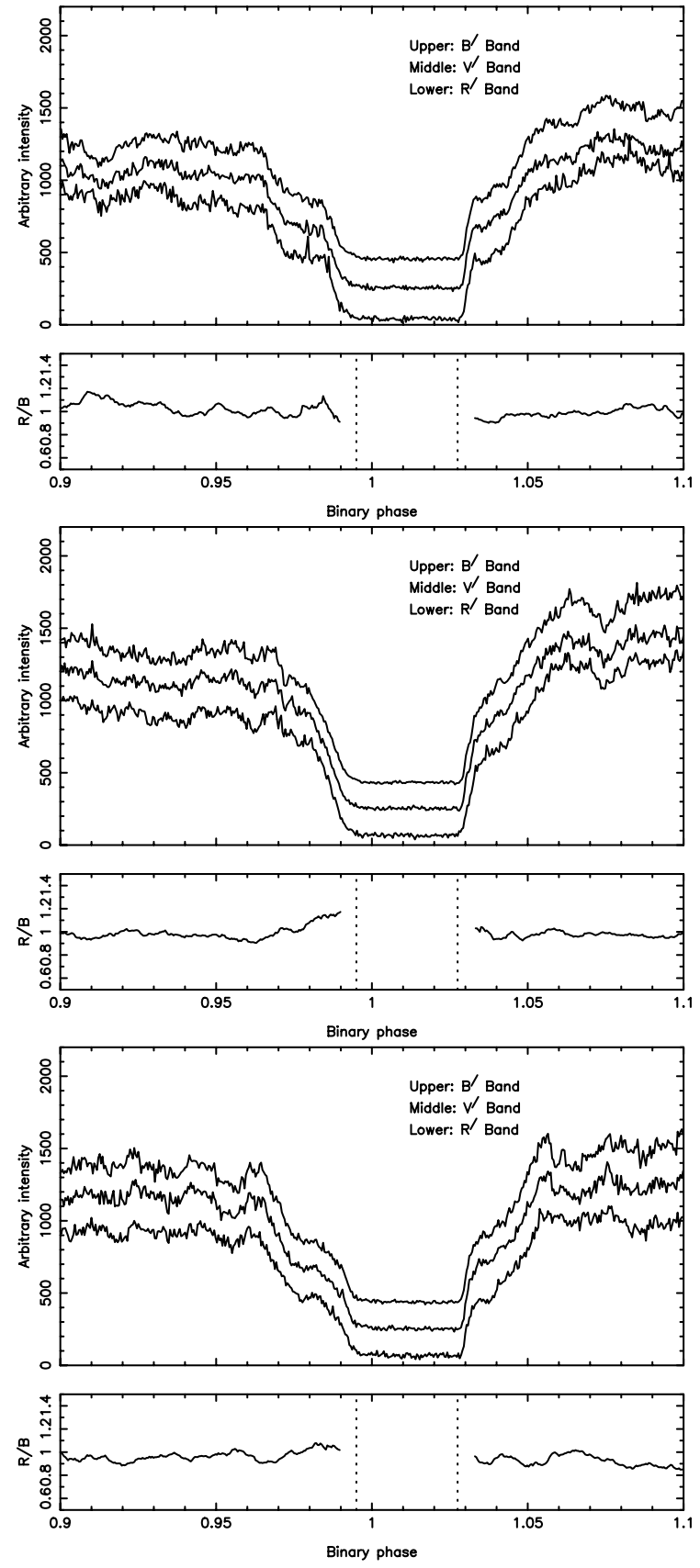

Fig. 2. Energy-band selected lightcurves for the three eclipses in Fig. 1, with the ratio $R^{\prime} / B^{\prime}$ against phase plotted below each observation. The vertical dotted lines in the $R^{\prime} / B^{\prime}$ plots indicate the approximate start and end phases of the deep eclipse. Not only is the eclipse ingress and egress shape in general more sharply defined in the $R^{\prime}$ band data than the $B^{\prime}$ band, but there are significant differences between the three ratio plots.

\subsection{Spectral fitting}

We now examine the same data using the complimentary approach of model fitting to time-resolved spectra. Each complete eclipse observation was sliced into consecutive $25 \mathrm{~s}$ intervals, with a single spectrum created from each interval. The duration of the intervals was chosen as a compromise between time-resolution and $S / N$, and is short enough to probe spectral variations on the typical timescale of the 

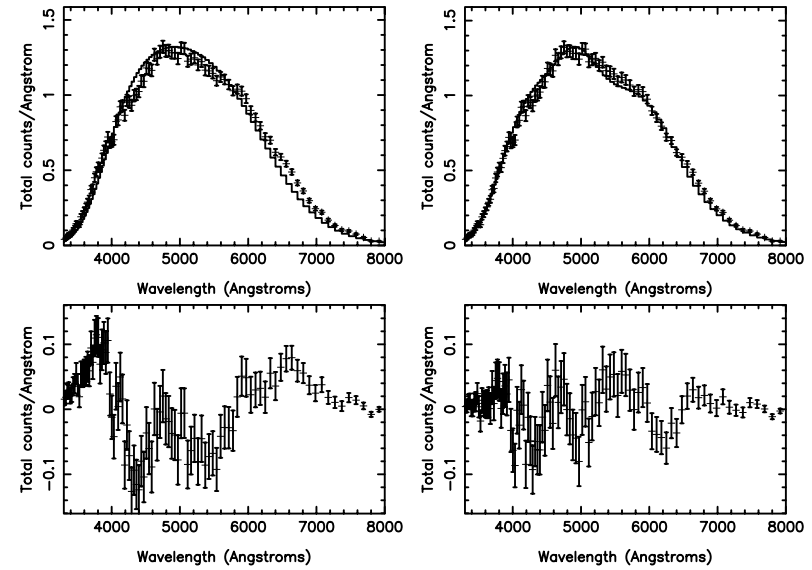

Fig. 3. Left: blackbody fit, with associated residuals, for the final $25 \mathrm{~s}$ slice in the first observation. Right: the same data, but now modelled with a blackbody and emission lines. The residuals are plotted on the same scale as in the left-hand box.

major ingress/egress features seen in the lightcurves. The spectra were corrected for electronic artifacts and pixel-to-pixel gain variations. Corresponding background spectra were obtained by using $150 \mathrm{~s}$ data intervals from the deepest part of the eclipse in all three observations, ensuring that any residual contribution from the donor star is removed from the spectrum, in addition to the sky.

Spectral fitting made use of the XSPEC model-fitting package (Arnaud 1996), developed for use in high-energy astrophysics, but adaptable to the analysis of low-resolving power optical data such as S-Cam spectra. In the left-hand panels of Fig. 3, we show the result of fitting a blackbody model against a post-eclipse slice (the final $25 \mathrm{~s}$ interval) from the first observation, together with the associated residuals. The optical flux therefore originates from the white dwarf, the accretion region and the accretion stream. The reduced $\chi^{2}$ for this fit was 4.8 . The systematic structure in the residuals clearly indicates the inadequacy of this single-component model.

In the right-hand panels of Fig. 3, we show the improvement to the fit by the addition of emission lines to the blackbody model. We fixed the blackbody temperature to be $10000 \mathrm{~K}$ which was the mean value derived after experimenting with $k T_{\mathrm{bb}}$ as a free parameter (the range in $k T_{\mathrm{bb}}$ was a few $1000 \mathrm{~K}$ ). The lines included in our fit were $\mathrm{H} \alpha$ and $\mathrm{H} \beta$, both of which are seen in emission in the data of Barwig et al. (1994), Silber et al. (1994), Ferrario et al. (1995) and Simic et al. (1998), and He I 5875, observed strongly in emission in many AM Her spectra, including V2301 Oph (Barwig et al. 1994). He II 4686 is also likely to be a strong feature of the spectrum. This line, however, lies sufficiently close to $\mathrm{H} \beta$ that, with our limited resolution, the two lines are blended. While our fit statistics show that it is necessary to have an emission component in this part of the spectrum, we are more than likely modelling the contribution of two lines with our single component labeled " $\mathrm{H} \beta$ ".

Using the published optical data of Simic et al., we estimated that the width $(\sigma)$ of $\mathrm{H} \alpha$ is $\sim 170 \AA$. The line widths in our model were therefore fixed at this value, and the

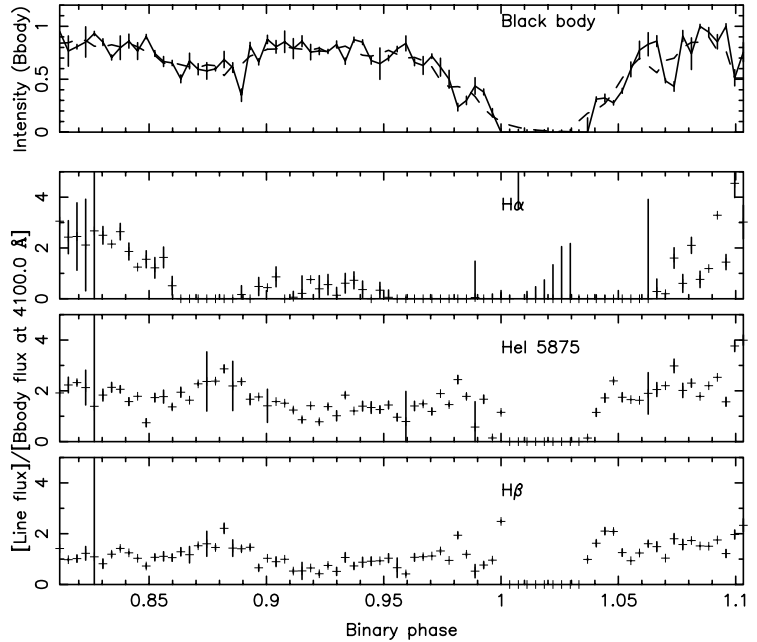

Fig. 4. Continuum and line strengths in observation 1. From top to bottom: phase-resolved, normalised line intensities for the following model components: blackbody (for $T_{\text {eff }}=10000 \mathrm{~K}$ ), $\mathrm{H} \alpha, \mathrm{He}$ I 5875 , and $\mathrm{H} \beta$. The line strengths are normalised to the instantaneous blackbody intensity, and refer to the height of the line as a fraction of the continuum height at $4130 \AA$ A. The final model line, the Broad Emission Component, is not plotted, since its identification is uncertain and it varied erratically with time.

centroid wavelengths were fixed at their laboratory values. We also added a "Broad Emission Component" centered on $3540 \AA$. This is to take into account the many features expected to exist to the blue side of He II 4686, including the Balmer limit, which it would be impractical to fit as individual components given our limited resolution. The width of this component was allowed to vary, but for the other lines the only adjustable parameter was the normalisation. With all model parameters present, the mean reduced $\chi^{2}$ over the first eclipse observation was 1.67 , compared to 6.0 for the blackbody alone.

In Fig. 4 we show the variations in line strengths with binary phase for the first eclipse observations. The mean $\chi^{2}$ for the second and third observations is worse than for the first: increasing to 1.9 and 2.4 respectively. This is probably due to long-term drifts in the gain, after warming and cooling of the cryogenic system.

The top panel of Fig. 4 shows the variation in the normalisation of the blackbody, together with a smoothed version of the same trend. It is seen that this curve closely resembles the shape of the white light lightcurve: this is expected since most of the photons detected will come from the continuum rather than the lines.

The trends in the three narrow lines in the model are plotted below the blackbody normalisation on the same time axis. In order to make the line strengths meaningful, we normalised them to the blackbody continuum strength: the plotted intensities represent the height of the line above the continuum, as a fraction of the continuum height at $4130 \AA$ A. If the line intensities are proportional to the underlying blackbody normalisation therefore, the resultant trend will be approximately flat. This is seen to be the case for He I 5875 and $\mathrm{H} \beta$. $\mathrm{H} \alpha$, however, becomes progressively fainter relative to the above lines, almost vanishing around $\phi=0.95$. It is likely that $\mathrm{H} \beta$ 


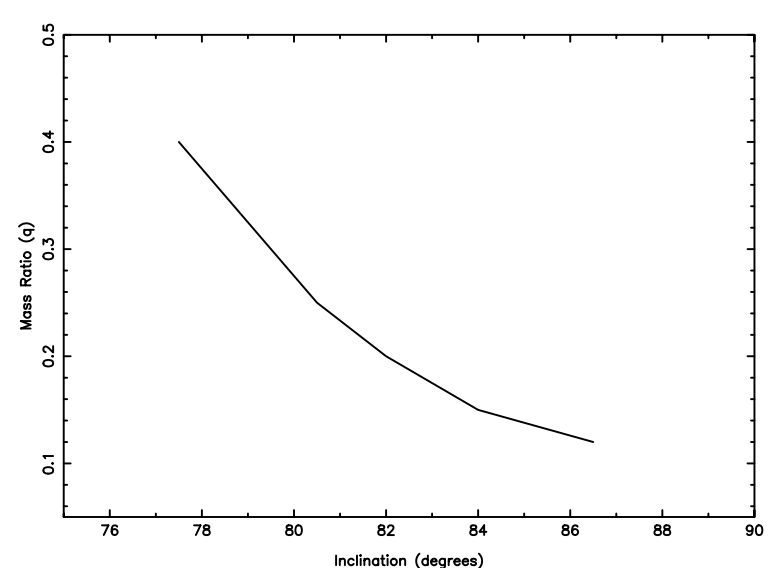

Fig. 5. The relationship between mass ratio $(q)$ and inclination for V2301 Oph, as defined by the eclipse geometry. The phase of the stream visibility favours $q=0.15, i=84.0^{\circ}$.

also becomes weaker, but that this is masked by the contribution from He II 4686 (see above). One physical reason for the Balmer lines to become weaker as the phase nears eclipse is that they are more likely to originate from the heated face of the secondary star (and hence not visible at eclipse), while the He II 4686 line originates from the accretion stream, which is visible for longer. This also explains why $\mathrm{H} \alpha$ is also somewhat slow to reappear after the sharp rise in continuum intensity during the egress, displaying a $\sim 0.02$ lag in phase. The final model parameter, the Broad Emission Component at $3540 \AA$ varies erratically, but is generally stronger in the post-eclipse phases than prior to the eclipse.

\section{Discussion}

In Sect. 3 we showed that the optical emission from V2301 Oph has a larger contribution from the accretion stream compared to many other polars. In order to explore this further, we trace the Roche potential out of the binary system along the line of sight from any point within the vicinity of the white dwarf. We then adjust the system parameters, (mass ratio $(q)$ and inclination $(i)$, and accretion spot location), under the constraints of grazing contact of this line of sight with the Roche lobe at particular phases. We initially adopted $(q, i)$ values of Steiman-Cameron \& Imamura (1999), namely 0.25 and $81.75^{\circ}$ respectively. However, with the usual assumption that the stream follows a ballistic and then a magnetic trajectory, we found that the stream would be fully eclipsed by $\phi=0.99$, whereas our observations show that the stream remains visible until phase $\phi \sim 0.995$. In order to resolve this, the donor star must become smaller, with a corresponding increase in the inclination. There are no individual constraints on $(q, i)$, only the pairs set by the eclipse length, as illustrated by the curve in Fig. 7. The choice of $q=0.15, i=84.0^{\circ}$ is sufficient to ensure stream visibility at phase 0.99 , so in the absence of further constraints we base our subsequent discussion on these values.

In Fig. 6, we show the orientation of the binary at different eclipse phases, for two different configurations of the threading region. The parameters used to generate these plots, apart from $(q, i)$, are a spot azimuth of $65^{\circ}$ and the magnetic field aligned at $20^{\circ}$ to the white dwarf spin axis, both of which are in line with the values in Steiman-Cameron \& Imamura (1999). The threading of material onto the magnetic field lines takes place between $0.2-0.12 a$ (where $a$ is the binary separation) for the set of plots on the left, and between $0.4-0.12 a$ for the set of plots on the right.

The scenario on the left can then be related to the eclipse lightcurve seen in the first observation (Fig. 1). By $\phi=0.967$, the white dwarf is fully eclipsed. This causes only a small dip in the lightcurve, however, and is only really apparent in the first observation. By $\phi=0.973$, the bright part of the magnetic stream closest to the white dwarf has also been eclipsed. The intensity then declines only gradually until $\phi=0.985$, when the threading region begins to be occulted. Brightness declines rapidly, and by $\phi=0.995$ the stream is entirely eclipsed.

On egress, the first component to emerge is the white dwarf, accounting for the sudden, steep rise in intensity. This is followed immediately by the hot stream, characterised by a slightly shallower slope. There is then another plateau as the empty mid-arch of the magnetic field is revealed. At $\phi=1.035$ the threading region again begins to emerge, and by $\phi=1.054$ the threading region is completely visible. The implication of this observation is that around one third of the stream brightness is in the region around the white dwarf, while the remaining two thirds arises in the threading region.

Turning to the scenario on the right, an increase in the extent of the threading region can explain the eclipse shape of the second observation. At $\phi=0.973$, the threading region is already starting to be eclipsed, and by $\phi=0.985$ is substantially hidden. However, the mid-arch of the magnetic field contains more material and is visible for longer. This accounts for the longer, brighter decline to eclipse seen in the second observation. As before the stream is fully eclipsed by $\phi=0.995$. At $\phi=1.054$, during the egress, the threading region is still not completely revealed, and hence the lightcurve continues to increase in brightness beyond this phase, in contrast to the first observation.

We therefore have a self-consistent explanation for the white-light variations seen in the first two observations, within the context of the standard ballistic-plus-magnetic stream. Almost all of the observed light arises in the stream, and two thirds of that is contributed by the threading region. Observation three closely resembles observation one, except that the threading region appears somewhat less bright.

The colour information, as presented in Sect. 4.1, also supports the above interpretation. Figure 2 shows that until the stream becomes rather faint at $\phi=0.99$, the second observation is redder than the first. This shows that what we are seeing in the second observation is cooler material in the furthest reaches of the threading region, whereas in the first observation, we are seeing material plunging deep into the field before threading. Observation three (in which there was a slight reddening compared to the first observation) is a little harder to understand, but the threading region is fainter here anyway, so is perhaps smaller and cooler than in the first observation. 


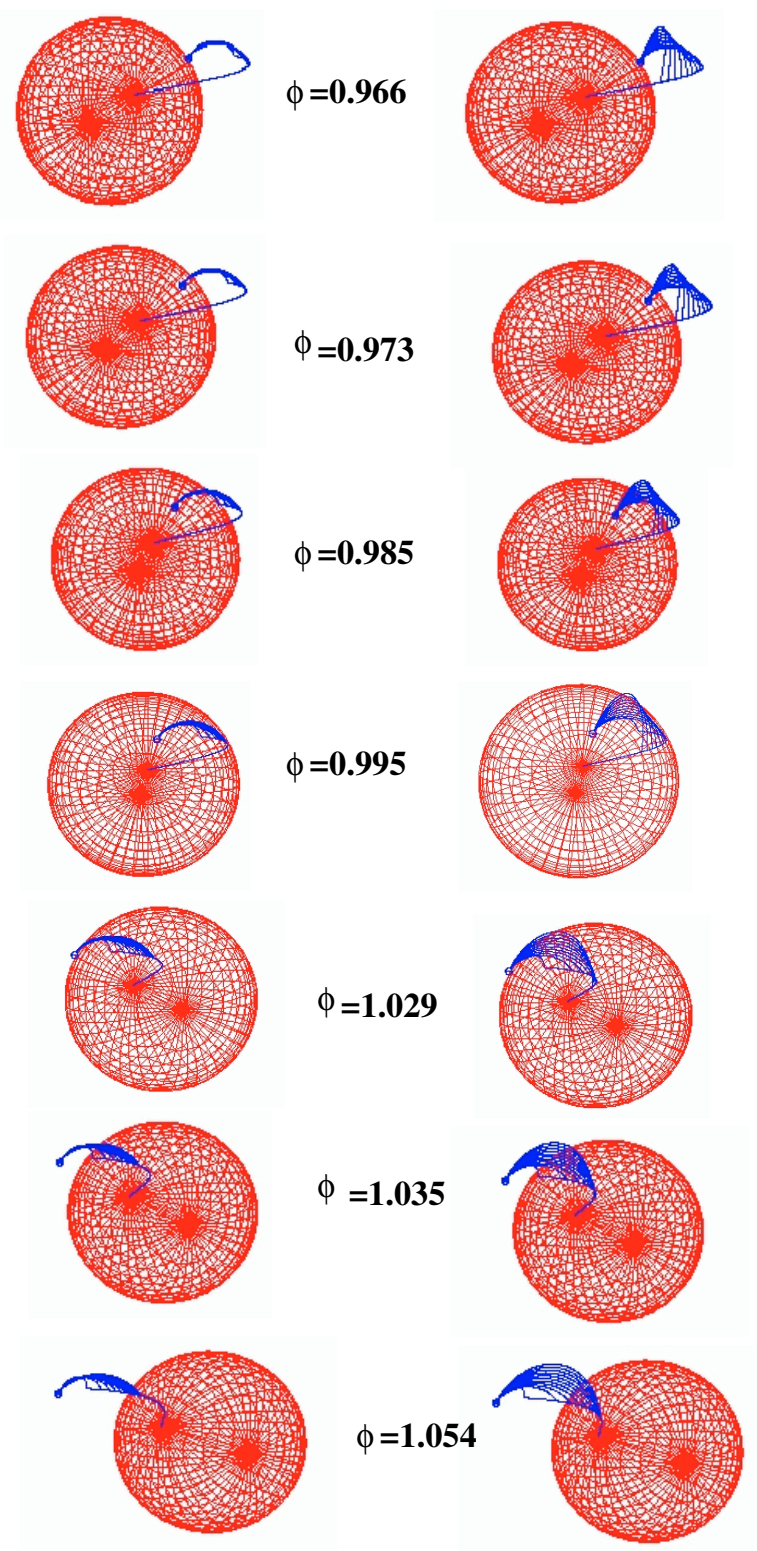

Fig. 6. The eclipse geometry of V2301 Oph, for $q=0.15, i=84^{\circ}$. The spot azimuth is $60^{\circ}$, and the magnetic field is aligned at $20^{\circ}$ to the white dwarf spin axis. The plots on the left show six phases of the eclipse for the case where the threading occurs later in the stream, while the plots on the right show the same six phases for the early threading scenario.

\section{Conclusions}

We have presented energy-resolved STJ photometry of the eclipsing polar V2301 Oph, reporting the first detection and analysis of stellar emission lines based on non-spectrally dispersed optical data. Three eclipses are observed in total, with significant variations in shape observed between them. These variations are shown to be consistent with the usual AM Her accretion scenario, whereby mass flows from the donor star to the white dwarf along a ballistic stream, before becoming threaded onto the magnetic field lines of the white dwarf. By varying the extent of the threading region along the ballistic stream, the major features of the observations can be accounted for, provided the mass ratio is decreased to 0.15 , with a corresponding increase in the inclination to $i=84.0^{\circ}$. The colour variations seen in the energy-resolved data are also consistent with this interpretation, since they suggest that the material is at its hottest when it penetrates most deeply into the magnetic field before becoming threaded.

These observations show that current STJ detectors can resolve optical emission lines. However, the resolution of such detectors is much poorer than can be obtained with dispersive spectrometers. Until now, nearly all STJ work has used the materials niobium and tantalum. The obvious next step is to migrate the technology to even lower critical-temperature superconductors such as hafnium $\left(T_{\mathrm{c}}=0.128 \mathrm{~K}\right.$, compared to $4.48 \mathrm{~K}$ for tantalum). With a band-gap well below a meV $(0.021 \mathrm{meV})$, hafnium has the potential to provide better energy resolution at all energies and is therefore of keen interest, for both visible and X-ray applications. The predicted tunnel-limited resolving power of hafnium is $\sim 64$ at $1 \mathrm{eV}$, so that the wavelength resolution is $\sim 2 \AA$ at Ly $\alpha, \sim 50 \AA$ at $5000 \AA$, and $150 \AA$ at $20000 \AA$.

Acknowledgements. We thank the ING staff at the WHT on La Palma for their excellent technical support during all the S-Cam2 telescope campaigns. The other members of the optical STJ development team at ESTEC are also thanked for their work on the S-Cam program.

\section{References}

Arnaud, K. A. 1996, Astronomical Data Analysis Software and Systems V, ed. G. Jacoby, \& J. Barnes, ASP Conf. Ser., 101, 17

Barwig, H., Ritter, H., \& Barnbantner, O. 1994, A\&A, 288, 204

Bridge, C. M., Cropper, M., Ramsay, G., et al. 2002, MNRAS, 336, 1129

Bridge, C. M., Cropper, M., Ramsay, G., et al. 2003, MNRAS, 341, 863

de Bruijne, J. H. J., Reynolds, A. P., Perryman, M. A. C., et al. 2002, A\&A, 381, L57

Ferrario, L., \& Wehrse, R. 1999, MNRAS, 310, 189

Ferrario, L., Wickramasinghe, D., Bailey, J., \& Buckley, D. 1995, MNRAS, 273, 17

Perryman, M. A. C., Favata, F., Peacock, A., et al. 1999, A\&A, 346, L30

Perryman, M. A. C., Cropper, M., Ramsay, G., et al. 2001, MNRAS, 324, 899

Rando, N., Peacock, A., Andersson, S., et al. 1998, Proc. SPIE, 3435, 74

Rando, N., Verveer, J., Verhoeve, P., Peacock, A., et al. 2000, Proc. SPIE, ATI-2000, Munich, in press

Reynolds, A. P., de Bruijn, J. H. J., Perryman, M. A. C., et al. 2003, A\&A, 4001209

Schmidt, G. D., \& Stockman, H. S. 2001, ApJ, 548, 410

Silber, A. D., Remillard, R. A., Horne, K., \& Bradt, H. V. 1994, ApJ, 424, 955

Simic, D., Barwig, H., Bobinger, A., et al. 1998, A\&A, 329, 115

Steeghs, D., Perryman, M. A. C., Reynolds, A., et al. 2003, MNRAS, 339,810

Steiman-Cameron, T. Y., \& Imamura, J. N. 1999, ApJ, 515, 404 